\title{
Two-dimensional photonic crystal slab with embedded silicon nanocrystals: Efficient photoluminescence extraction
}

Lukáš Ondič, Marian Varga, Karel Hruška, Alexander Kromka, Kateřina Herynková, Bernd Hönerlage, and Ivan Pelant

Citation: Appl. Phys. Lett. 102, 251111 (2013); doi: 10.1063/1.4812477

View online: https://doi.org/10.1063/1.4812477

View Table of Contents: http://aip.scitation.org/toc/apl/102/25

Published by the American Institute of Physics

\section{Articles you may be interested in}

Nanocrystalline diamond photonics platform with high quality factor photonic crystal cavities

Applied Physics Letters 101, 171115 (2012); 10.1063/1.4764548

Luminescence of free-standing versus matrix-embedded oxide-passivated silicon nanocrystals: The role of matrix-induced strain

Applied Physics Letters 101, 143101 (2012); 10.1063/1.4756696

Active planar optical waveguide made from luminescent silicon nanocrystals

Applied Physics Letters 82, 955 (2003); 10.1063/1.1544433

\section{PHYSICS TODAY}

MANAGER'S GUIDE

WHITEPAPERS

\section{READ NOW}

PRESENTED BY

Multiphysics Simulation 


\title{
Two-dimensional photonic crystal slab with embedded silicon nanocrystals: Efficient photoluminescence extraction
}

\author{
Lukáš Ondič, ${ }^{1,2,3, a)}$ Marian Varga, ${ }^{1}$ Karel Hruška, ${ }^{1}$ Alexander Kromka, ${ }^{1}$ \\ Kateřina Herynková, ${ }^{1}$ Bernd Hönerlage ${ }^{3}$ and Ivan Pelant ${ }^{1}$ \\ ${ }^{1}$ Institute of Physics, Academy of Sciences of the Czech Republic, v.v.i., Cukrovarnická 10, 162 53, Prague 6, \\ Czech Republic \\ ${ }^{2}$ Faculty of Mathematics and Physics, Charles University, Ke Karlovu 3, 121 16, Prague 2, Czech Republic \\ ${ }^{3}$ IPCMS-DON Unité Mixte, UMR 7504, CNRS-ULP, 23 rue du Loess, BP 43, 67034 Strasbourg Cedex 2, \\ France
}

(Received 23 May 2013; accepted 10 June 2013; published online 26 June 2013)

\begin{abstract}
A two-dimensional photonic crystal (PhC) slab was fabricated from a luminescent planar waveguide, formed by a $(800 \mathrm{~nm}$ thick) layer of silicon nanocrystals (SiNCs) embedded in a polished silica plate. Dimensions of the PhC were designed so that light emitted by SiNCs under excitation with an external UV source can, during its propagation in the layer, interact with the periodicity and be Bragg-diffracted into air. This approach leads to up to 8-fold vertical extraction enhancement of SiNCs luminescence from the $\mathrm{PhC}$ slab compared to the bare planar layer. Results of the experiment are supported by the computer simulation. (C) 2013 AIP Publishing LLC. [http://dx.doi.org/10.1063/1.4812477]
\end{abstract}

Silicon quantum dots or nanocrystals (SiNCs) sized a few nanometers in diameter are brightly fluorescent particles, which hold promise towards many potential applications: photonic light-emitting devices, biological sensors and labels, fluorescence markers, and tandem solar cells. ${ }^{1-3}$ When employed in solid photonic light sources, SiNCs are usually embedded in a transparent solid matrix, silicon dioxide $\mathrm{SiO}_{2}$ (silica) as a rule. Extraction of luminescence radiation, originating in SiNCs, from the device into air is then of fundamental importance, since the light output is limited by total internal reflection on the matrix-air interface. In other words, even high internal luminescence quantum yield may be annulled to a considerable extent due to total internal reflection losses. Here, two-dimensional patterning of the device active surface may help markedly. If the pattern is designed periodic in two-dimensions with a lattice constant of the order of luminescence wavelength, a two-dimensional (2D) photonic crystal $(\mathrm{PhC})$ is created on the surface. ${ }^{4}$ If dimensions of the periodicity are carefully chosen, guided light propagating in all planar directions can be Bragg-diffracted and leak into air under specific angles defined by photonic band diagram. 5 Guided modes are then referred to as leaky modes. ${ }^{7}$

Some preliminary reports on photoluminescence (PL) of SiNCs combined with PhC were already published by our group. ${ }^{8,9}$ We have already shown that shape of the emission spectrum of SiNCs can be controlled by depositing them on the surface of a 2D-PhC made of nanocrystalline diamond. ${ }^{9}$ However, only a modification of PL spectrum shape was achieved but no light extraction enhancement in a given direction was observed. The main reasons for that were the low quality of the photonic structure and/or insufficient overlap of spatial distribution of SiNCs with electric field of leaky modes.

In this letter, we report on experimental and theoretical investigation of silica 2D PhC slab with embedded SiNCs, prepared by advanced methods. We demonstrate that light

a)Electronic mail: ondic@fzu.cz emitted by SiNCs couples to leaky modes of the $\mathrm{PhC}$ and is efficiently extracted out into air in preferred directions.

The PhC sample was prepared by employing two main steps. First, SiNCs rich layer was created inside polished silica substrate using $\mathrm{Si}^{+}$-ion implantation, forming basically a planar waveguiding layer beneath the silica surface. Second, surface of the active layer was periodically patterned by employing electron beam lithography with subsequent reactive ion etching.

Implantation of $\mathrm{Si}^{+}$-ions $(400 \mathrm{keV}$, implant fluence of $1 \times 10^{17} \mathrm{~cm}^{-2}$ ) into a polished $1 \mathrm{~mm}$ thick silica plate followed by thermal annealing at $1100^{\circ} \mathrm{C}$ and forging lead to formation of about $800 \mathrm{~nm}$ thick SiNCs-rich luminescent layer below the silica surface. Details of the preparation method can be found in Ref. 10. Spatial distribution of SiNCs can be approximated by an asymmetric Gaussian function with maximum located closer to the pure $\mathrm{SiO}_{2}$ layer (see blue line in Fig. 1(a) depicting the refractive index distribution of the sample which follows the spatial distribution of SiNCs). Under external continuous wave (cw) UV laser $(325 \mathrm{~nm})$ excitation at room temperature, the main PL emission band is located in the red spectral region peaking at around $780 \mathrm{~nm}$ (Fig. 1(b), black curve). Physical origin of this emission is attributed to SiNCs with diameter of 4-5 nm (detected by Raman scattering ${ }^{10}$ ), but there exist experimental and theoretical studies indicating that also very small SiNCs may play an important role in PL mechanism. ${ }^{11}$

For creating a periodical pattern etched on top of the SiNCs-rich layer we adapted our method used previously for fabrication of $\mathrm{PhC}$ on a nanocrystalline diamond layer. ${ }^{12}$ $2 \mathrm{D}-\mathrm{PhC}$ consisting of columns arranged to a square symmetry was chosen in order to obtain a structure that can interact with guided modes propagating in all planar directions. First the implanted silica plate was cleaned by ultrasonication in acetone for $10 \mathrm{~min}$ and dried by a nitrogen gun. Then it was coated with a positive electron sensitive polymethyl methacrylate (PMMA) polymer resist of $120 \mathrm{~nm}$ in thickness. Electron 


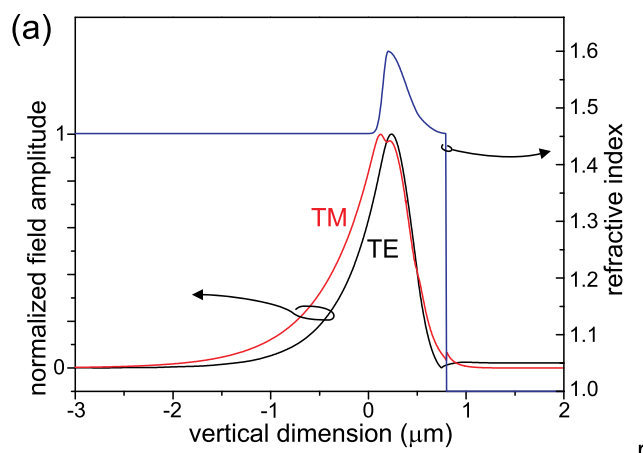

(b)

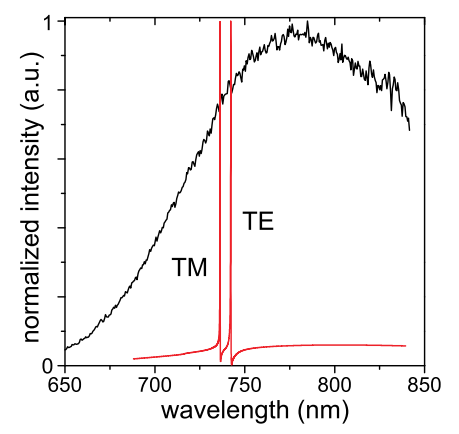

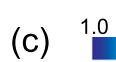

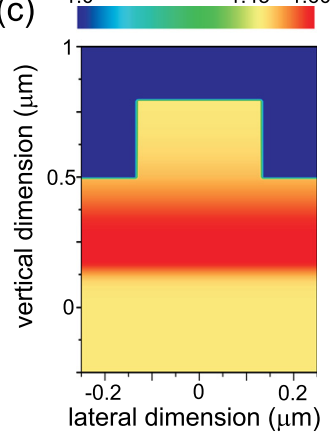

FIG. 1. (a) Blue curve depicts refractive index profile of the implanted silica plate in the vertical direction which follows the spatial distribution of SiNCs. Position " 0 " is at the interface of pure silica and SiNCs-rich layer. Simulated profiles of electric field amplitudes of $\mathrm{TE}_{0}$ and $\mathrm{TM}_{0} \mathrm{PhC}$ leaky modes at the $\Gamma$-point, whose spectral positions are shown in (b), are plotted by black and red curves, respectively. $\mathrm{E}_{y}$ component (in the sample plane) of the TE and $\mathrm{E}_{z}$ component (normal to the sample plane) of the TM leaky mode are plotted. The intensity is normalized to unity. (b) Plot of the normalized PL emission spectrum of SiNCs embedded in $\mathrm{SiO}_{2}$ plate compared to the simulated TE and TM leaky resonances of the $\mathrm{PhC}$ at the $\Gamma$-point of the photonic band diagram. (c) Vertical cut of the 3D-computation domain (zoomed in the $\mathrm{z}$-direction) through the middle of the $\mathrm{PhC}$ column.

beam lithography was used to create holes into the polymer with a diameter of approximately $300 \mathrm{~nm}$, ordered into a square lattice with a lattice constant $\Lambda \approx 500 \mathrm{~nm}$. Then, a $70 \mathrm{~nm}$ thick golden layer was evaporated on the polymer filling the holes. Afterwards, lift-off of the resist was performed by ultrasonication in acetone to form a mask for the subsequent plasma etching. Capacitively coupled reactive ion etching in a pure $\mathrm{SF}_{6}$ gas mixture was applied to form geometrically ordered columns covering the surface area of $1 \times 1 \mathrm{~mm}^{2}$ placed in the middle of the sample. Remaining $\mathrm{Au}$ mask was removed by wet etching. Columns featuring long range periodicity and having well defined diameter $d \approx 265 \mathrm{~nm}$ and height $h \approx 300 \mathrm{~nm}$ were obtained as shown in SEM images of the final PhC structure in Fig. 2.

Dimensions of the $\mathrm{PhC}$ were designed with the goal to obtain high extraction efficiency of light emitted by SiNCs and trapped in the layer and also to test a possible lasing effect that can occur due to the existence of modes with low group velocity (the so-called slow modes). ${ }^{13,14}$ Therefore, it was desirable to obtain spectral overlap of the PhC leaky modes with the emission spectrum of SiNCs. Moreover, as the extraction is most efficient in the direction normal to the sample plane (z-direction) and also the slow modes are diffracted in this direction, we attempted to obtain overlap of the PL emission spectrum with leaky resonances located at the $\Gamma$-point of photonic band diagram.

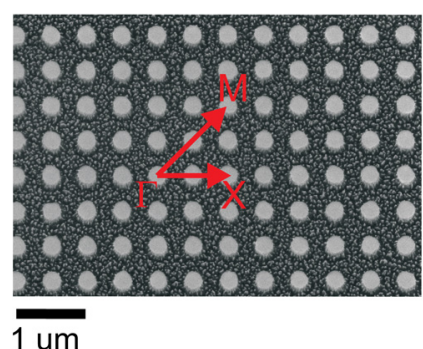

$1 \mathrm{um}$

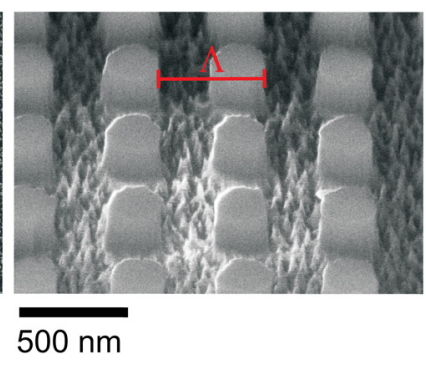

FIG. 2. SEM images of the final 2D-PhC structure with the $500 \mathrm{~nm}$ lattice constant $\Lambda$, columns diameter of $265 \mathrm{~nm}$, and height of $300 \mathrm{~nm}$. Directions of the high symmetries of the square lattice Brillouin zone are depicted in the left image.

Photonic band diagram, as dispersion of photonic modes supported by the device, typically shows the modal frequency as a function of the wavevector, or in other representation, it plots modal air/vacuum wavelengths against the propagation angles in air. To simulate the problem, we used RCWA method (RSoft DiffractMOD) which allows to compute the transmission and/or reflection spectra of periodic dielectric media and locate leaky resonances which are revealed in the transmission/reflection spectra as narrow minima/maxima. ${ }^{15,16}$ Refractive index in the SiNCs-rich layer was characterized by an asymmetric Gaussian function with maximum value equal to 1.6 (Fig. 1(a), blue curve). ${ }^{10}$ A cut through the middle of the computation domain, zoomed in the z-direction, is shown in Fig. 1(c). Details of the simulation can be found in supplementary material. ${ }^{17}$ By varying the lattice constant $\Lambda$, diameter $d$, and height of columns $h$ we chose a suitable combination of the parameters used as input data for lithographic fabrication. The final dimensions of the prepared $\mathrm{PhC}$, except the lattice constant, slightly $( \pm 10 \mathrm{~nm})$ changed during the preparation process due to the great number of steps involved; however, the overlap of the simulated $\mathrm{TE}_{0}$ and $\mathrm{TM}_{0}$ leaky modes (extracted in direction normal to the sample plane) with SiNCs emission spectrum was preserved as it is shown in Fig. 1(b) (dimensions obtained from the SEM images of the final $\mathrm{PhC}$ were used in the presented result of the simulation).

2D maps of electric field amplitude distribution of the two leaky modes are shown in supplementary material. ${ }^{17}$ Vertical cuts taken near the maximum of the modes and showing profile of the electric field amplitudes of the $E_{y}$ component of $\mathrm{TE}_{0}$ and $E_{z}$ component of the $\mathrm{TM}_{0}$ leaky modes at the $\Gamma$-point are shown in Fig. 1(a) with respect to the refractive index profile. It is evident that modes maxima are located in the SiNCs-rich layer. We can thus expect that light emitted by SiNCs will couple to these leaky modes, thanks to both their good spectral overlap with SiNCs PL spectrum and spatial overlap with SiNCs distribution.

In order to test performance of the $\mathrm{PhC}$ device, we measured the PL emission intensity as a function of the detection angle with respect to sample normal. The sample was excited through the silica substrate with a $325 \mathrm{~nm} \mathrm{cw}$ laser beam focused on the PhC. The PL was collected from the front side of the sample by an optical fiber (collection half-angle $1^{\circ}$ ) attached to a rotating arm, allowing to perform angle-resolved measurements. The sample was placed in a rotational holder which enabled us to investigate leaky modes along both the $\Gamma-\mathrm{M}$ and $\Gamma-\mathrm{X}$ square symmetry directions. Results of these 

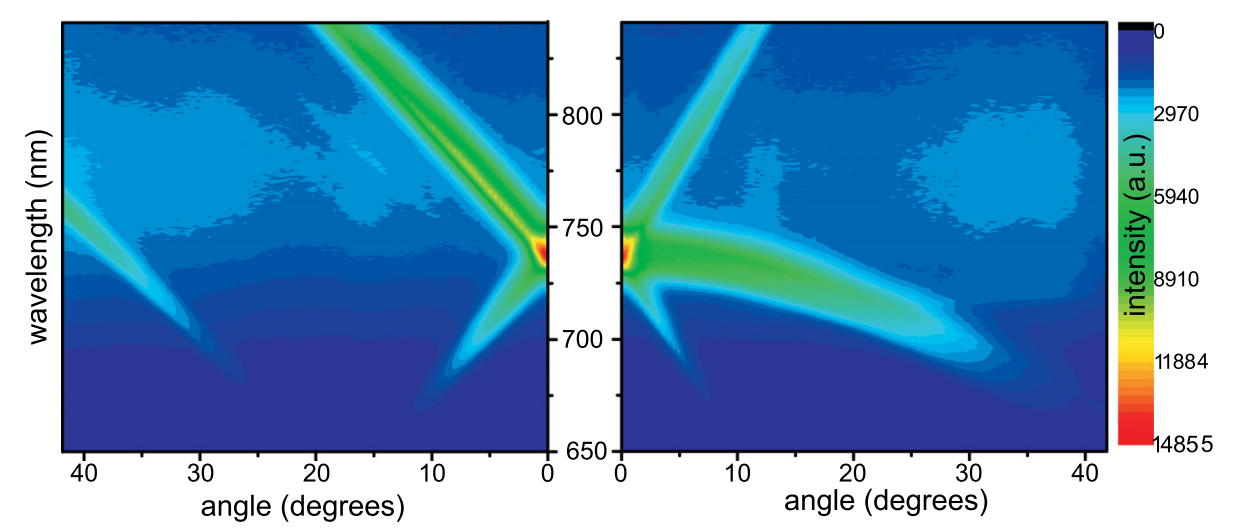

FIG. 3. PL emission spectra of SiNCs embedded in $\mathrm{SiO}_{2}$ plate with 2D-PhC measured along the $\Gamma$-M (left) and $\Gamma$-X (right) crystal directions as a function of the detection angle. Angle $\theta=0^{\circ}$ is normal to the sample surface.

measurements are summarized in 2D maps of PL emission intensities (Fig. 3) where the angle $\theta=0^{\circ}$ is normal to the sample surface. Relatively narrow peaks several times more intense than the spectrally broad background are superimposed on the broad spectra, typical for the uncorrugated sample (broad band is clearly visible for the larger detection angles). These narrow peaks enhance the PL extraction efficiency in the given directions and cause that the light emission is strongly directional.

The physical mechanism underlying the PL extraction enhancement process, already briefly mentioned in the introduction, is as follows. In the case of a planar uncorrugated layer, light emitted from SiNCs and heading towards the airsample interface is either coupled to the guided modes of the layer or it radiates out of the sample if it lies inside the air lightcone defined by the angle of total internal reflection. The latter is responsible for the broad PL emission band. However, when the $\mathrm{PhC}$ columns are introduced on the top, guided modes can now interact with the periodicity and in case they fulfill the Bragg diffraction condition, they are diffracted into air under the specific angles defined by the Bragg condition. Thus, they serve as efficient radiative channels for light previously "trapped" in the layer. These radiative modes are the leaky modes and in the angle-resolved PL spectra they form photonic bands as it is visible also in result of our experiment. It is also clear that the extraction of light is most efficient for small detection angles (close to the sample normal, see Fig. 3) due to degeneracy of leaky modes at the $\Gamma$-point. ${ }^{18}$

For the case of vertical emission, PL spectra of the $\mathrm{PhC}$ compared to the unpatterned layer of SiNCs in silica plate are plotted in Fig. 4(a) (red vs black curve). As explained above, the narrow peak superimposed on the broad spectra represents emission from SiNCs extracted via leaky modes. High enhancement of extraction efficiency by a factor of 8 was achieved for the maximum located at $736 \mathrm{~nm}$ (Fig. 4(a), dotted blue curve). The leaky mode band can be decomposed into two Lorentzian peaks with maxima at 736 and $745 \mathrm{~nm}$ (Fig. 4(b)), the first one being TM and the second one TE resonance. The Q-factors, obtained from the widths of the Lorentzian fits, are 37 and 43 for the TM and TE resonance, respectively. These low Q-factors are the reason for the high extraction efficiency; light propagates for a short time only in the layer, which minimizes optical losses, and is quickly extracted out to air. The agreement between the spectral positions of the simulated leaky resonances and the measured ones (Fig. 4(b)) is very satisfying. As discussed in detail later in connection with Fig. 4(c), the slight difference in spectral positions of the TE mode obtained here can be easily explained by the uncertainty of the refractive index value used in the simulation. However, the computed resonances are much more narrower (and thus Q-factor is much larger-of the order of 1000) than the measured counterparts. This difference is caused by the presence of optical losses (absorption, scattering on the sample imperfections) in the real sample which is not included in the simulation and which would lead to broadening of the resonances. Another reason for the broadened resonances may be that the PL collection half-angle is larger compared to the simulation, approximately $1^{\circ}\left(\theta=0^{\circ} \pm 1^{\circ}\right)$ and thus all modes outcoupled within this angle are detected. In the simulation, only light
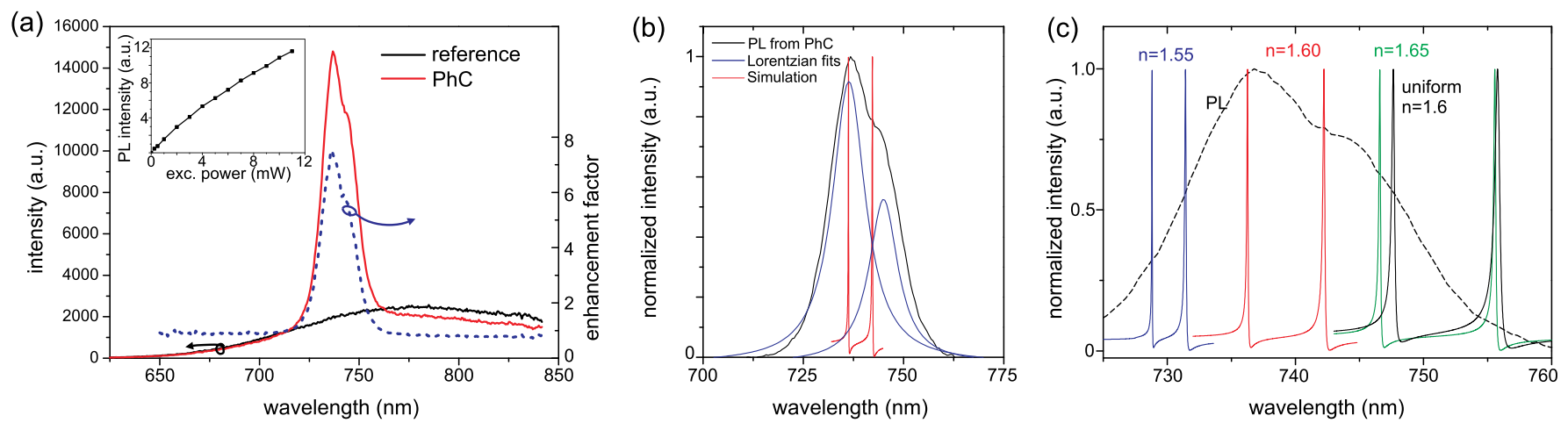

FIG. 4. (a) PL emission of SiNCs embedded in $\mathrm{SiO}_{2}$ plate with and without the 2D-PhC structure together with the PL extraction enhancement curve computed as ratio of the two PL curves. Inset shows the intensity of the PhC PL maximum (maximum of the leaky resonance) as a function of the excitation power. (b) PL emission band of the PhC with subtracted broad background showing only the leaky band fitted with two Lorentzian peaks, compared with the simulated $\mathrm{TE}$ and TM leaky resonances of the PhC. (c) PhC leaky resonances simulated for different values of refractive index maximum of SiNCs-rich layer. 
outcoupled exactly in direction normal to the sample plane $\left(\theta=0^{\circ}\right)$ is considered.

In order to check possible lasing effect for the slow modes at the $\Gamma$-point, the experiment in which the excitation power was gradually increased, was carried out for the normal detection. However, the dependence of emission intensity at the leaky mode peak maximum on the excitation power (inset of Fig. 4(a)) does not show any super-linear behavior typical when the lasing threshold is achieved.

It should be finally noted that the spectral position of the leaky resonances at the $\Gamma$-point is very sensitive to the spatial profile and absolute value of the $\mathrm{PhC}$ refractive index as shown in Fig. 4(c). Decreasing value of the refractive index maximum (from 1.65 to 1.55 ) spectrally blue-shifts position of the leaky resonances and also brings TE and TM resonances closer to each other. Also, the difference between uniform and asymmetric Gaussian refractive index profiles of SiNCs-rich layer is demonstrated for the value of index equal to 1.6. Leaky modes are spectrally red-shifted when uniform index distribution in SiNCs-rich layer is supposed. This shows the importance of taking into consideration the Gaussian profile when performing the simulations.

To summarize, we demonstrated both experimentally and via modelling that the $2 \mathrm{D}-\mathrm{PhC}$ structure is able to enhance extraction efficiency of PL originating in SiNCs as much as 8-times for the case of vertical emission and remains considerably high even for other emission angles. Engineering of the refractive index value and profile can lead to further increase of the enhancement factor, which may be beneficial for future photonic light sources based on silicon nanoparticles.

This work was supported by the GAUK (Grant SVV2013-267306) and GACR (P108/12/G108). This work was carried out in frame of the LNSM infrastructure and was also supported by the scholarship "thèse en cotutelle" of the Ministry of Education of France (L.O.). We thank S. Cheylan for providing the ion-implanted silica plates.

${ }^{1}$ Silicon Nanophotonics: Basic Principles, Current Status and Perspectives, edited by L. Khriachtchev (Pan Stanford Publishing, 2009).

${ }^{2}$ Device Applications of Silicon Nanocrystals and Nanostructures, edited by N. Koshida (Springer, 2009).

${ }^{3}$ Silicon Nanocrystals, edited by L. Pavesi and R. Turan (Wiley-VCH, 2010).

${ }^{4}$ J. D. Joannopoulous, S. G. Johnson, J. N. Winn, and R. D. Meade, Photonic Crystals: Molding the Flow of Light (Princeton University Press, Princeton, 2008).

${ }^{5}$ J. J. Wierer, A. David, and M. M. Megens, Nature Photon. 3, 163 (2009).

${ }^{6}$ C. Wiesmann, K. Bergenek, and U. T. Schwarz, Laser Photonics Rev. 3, 262 (2009).

${ }^{7}$ S. Fan and J. D. Joannopoulos, Phys. Rev. B 65, 235112 (2002).

${ }^{8}$ P. Janda, J. Valenta, J.-L. Rehspringer, R. R. Mafouana, J. Linnros, and R. G. Elliman, J. Phys. D 40, 5847-5853 (2007).

${ }^{9}$ L. Ondič, O. Babchenko, M. Varga, A. Kromka, J. Čtyroký, and I. Pelant, Sci. Rep. 2, 914 (2012).

${ }^{10}$ T. Ostatnický, J. Valenta, I. Pelant, K. Luterová, R. Elliman, S. Cheylan, and B. Hönerlage, Opt. Mater. 27, 781 (2005).

${ }^{11}$ L. Khriachtchev, S. Ossicini, F. Iacona, and F. Gourbilleau, Int. J. Photoenergy 2012, 872576.

${ }^{12}$ L. Ondič, K. Dohnalová, M. Ledinský, A. Kromka, O. Babchenko, and B. Rezek, ACS Nano 5, 346 (2011).

${ }^{13}$ K. Sakoda, Opt. Express 4, 167 (1999).

${ }^{14}$ L. Ondič and I. Pelant, Opt. Express 20, 7071 (2012).

${ }^{15}$ D. Rosenblatt, A. Sharon, and A. A. Friesem, IEEE J. Quantum Electron. 33, 2038 (1997).

${ }^{16}$ N. Ganesh, W. Zhang, P. C. Mathias, E. Chow, J. A. N. T. Soares, V. Malyarchuk, A. D. Smith, and B. T. Cunningham, Nat. Nanotechnol. 2, 515 (2007).

${ }^{17}$ See supplementary material at http://dx.doi.org/10.1063/1.4812477 for additional information about the computer RCWA simulation.

${ }^{18}$ N. Ganesh, I. D. Block, P. C. Mathias, W. Zhang, E. Chow, V. Malyarchuk, and B. T. Cunningham, Opt. Express 16, 21626 (2008). 